\title{
A RELAÇÃO ENTRE O PLANEJAMENTO E O SUCESSO DO PROJETO SOB OS EFEITOS DO RISCO E DA CAPACITAÇÃO PROFISSIONAL
}

\author{
THE RELATIONSHIP BETWEEN PLANNING AND PROJECT SUCCESS UNDER THE \\ EFFECTS OF RISK AND PROFESSIONAL TRAINING
}

\author{
Lucio Barbosa Roubach \\ Mestre em Administração \\ Universidade Federal do Espírito Santo - UFES. \\ Vitória, ES - Brasil. \\ lroubach@gmail.com \\ Marcos Paulo Valadares de Oliveira \\ Doutor em Administração \\ Universidade Federal do Espírito Santo - UFES \\ Vitória, ES - Brasil. \\ marcos.p.oliveira@ufes.br
}

\section{Resumo}

O planejamento é considerado um dos maiores colaboradores para o sucesso dos projetos. Pesquisas recentes dedicaram-se a estudar o comportamento dessa relação em diferentes contextos. Sugere-se que projetos expostos a maiores níveis de riscos demandem maior necessidade de planejar para alcançar o sucesso, enquanto projetos com maior número de profissionais capacitados apresentem menor necessidade de planejamento para alcançar o sucesso. Este trabalho buscou investigar a influência do planejamento no sucesso do projeto sob diferentes níveis de risco e de participação de profissionais capacitados em projetos no Brasil. Para tanto, foram avaliados os efeitos das moderações a partir da análise das respostas de 257 gerentes de projetos, por meio da Modelagem de Equações Estruturais. Os resultados mostram que o nível de risco modera o impacto do planejamento na dimensão de sucesso eficiência do projeto, enquanto a participação de profissionais capacitados não modera as relações do planejamento com qualquer dimensão de sucesso (eficiência ou eficácia). Foi possível deduzir que aprimorar o planejamento para alcançar o sucesso em eficiência do projeto é uma maneira que as empresas brasileiras dispõem para compensar o efeito dos riscos na relação. Em contrapartida, conclui-se que o fato de possuir uma equipe formada por grande parcela de colaboradores com capacitação não reduz a dependência da organização por planejar para alcançar o sucesso.

Palavras-chaves: Planejamento de projetos. Risco. Capacitação profissional. Sucesso de projetos.

\begin{abstract}
Planning is considered one of the greatest contributors to project success. Researchers have focused on the behavior of this relationship in various contexts. It is suggested that projects exposed to higher levels of risk require a greater need to plan to achieve success, while projects with a greater number of trained professionals demand less need for planning to achieve success. The present study investigates the influence of planning to project success under different risk levels and participation of trained professionals in Brazil's projects. Therefore, moderating effects based on the analysis of 257 project managers' responses were studied, through Structural Equations Modeling. The results show that the level of risk moderates the impact of the planning on the Project Efficiency success dimension, while the participation of trained professionals does not moderate the relationship between the planning and the project success in any success dimensions (efficiency or effectiveness). It was possible to deduce that improving the planning for achieving Project Efficiency is a manner that Brazilian companies follow to offset the impact of risks in the relationship. Regarding the participation of trained professionals, it is concluded that having a project team composed of many trained employees does not reduce the firm's planning dependence for achieving the success.
\end{abstract}

Keywords: Project planning. Risk. Professional training. Project success.

Cite como

American Psychological Association (APA)

Roubach, L. B., \& Oliveira, M. P. V. (2021, set./dez.). A relação entre o planejamento e o sucesso do projeto sob os efeitos do risco e da capacitação profissional. Revista de Gestão e Projetos (GeP), 12(3), 88-120. https://doi.org/10.5585/gep.v12i3.19048. 


\section{Introdução}

O alto nível de risco é percebido como um problema potencial nos projetos (PMI, 2013) e um obstáculo para o sucesso nas categorias de custo, tempo e desempenho técnico (Kerzner, 2009). Os padrões profissionais usualmente aceitos, como as boas práticas recomendadas no Project Management Body of Knowledge (PMBOK®) pelo Project Management Institute (PMI), enfatizam fortemente a importância de investir nos processos de gerenciamento de projetos para suportar o que foi planejado. A suposição por trás dessa posição é que o planejamento reduz as incertezas e aumenta a probabilidade de sucesso (Dvir, Tzvi, \& Shenhar, 2003).

Pesquisas indicaram o nível de risco percebido pelos gerentes de projetos como moderador da relação entre a qualidade do planejamento e o sucesso do projeto (Zwikael, Pathak, Singh, \& Ahmed, 2014; Zwikael \& Sadeh, 2007), ou seja, a percepção do risco possui relevância ao executar processos de gerenciamento de projetos na busca por seu êxito. Zwikael et al. (2014) destacou que, apesar de existirem vários trabalhos que considerem a qualidade do planejamento como fonte de sucesso e trabalhos que indicam o risco como variável moderadora dessa relação, é importante que pesquisas futuras se preocupem em comprovar a aplicabilidade desses mesmos efeitos em países distintos, dadas as diferentes condições de acesso e de disponibilidade à informação, às ferramentas, às técnicas de gerenciamento de projetos e ao mercado de trabalho, bem como na presença de fatores moderadores para essa relação orientados para os indivíduos.

É possível também identificar na literatura diversos estudos que destacam a relevância da capacitação dos profissionais envolvidos no projeto e indicam a sua relação com o desempenho (A. W. Brown, Adams, \& Amjad, 2007; Khan \& Rasheed, 2015; Lee-kelley \& Blackman, 2012; Tabassi \& Bakar, 2009). Por meio da Teoria do Capital Humano, onde o estoque de capital humano gera valor aos negócios da organização (A. W. Brown et al., 2007), e da aplicação desses conceitos na esfera do gerenciamento de projetos, sugere-se que diferentes demandas por profissionais capacitados nas equipes resultem em diferentes efeitos nos esforços para alcançar o sucesso e, portanto, na dependência por planejar as atividades, ou seja, quanto menor o número de profissionais capacitados 
para desempenhar suas as funções, maior será a necessidade de se dedicar atenção ao planejamento do projeto.

Com base nas informações apresentadas, o objetivo do artigo foi investigar a influência do planejamento no sucesso do projeto sob diferentes níveis de risco e de participação de profissionais capacitados em projetos no Brasil. Para tanto, foi elaborado modelo que relaciona duas importantes escalas de medida sob efeitos de moderação, servindo como um guia para a elaboração e teste das hipóteses que permitiram atender ao objetivo da pesquisa.

Como contribuições do estudo, ao final são ofertadas recomendações e orientações que direcionem os esforços dos gerentes de projetos em atuação ou profissionais do mercado, sobretudo no que diz respeito à fase de planejamento, sobre como melhor aplicar as ferramentas disponíveis e otimizar resultados dentro das características do projeto. Assim, a principal contribuição teórica advém da exploração do tema que cerca a participação de profissionais capacitados como fator significante em projetos.

\section{Fundamentação teórica}

Neste tópico, serão apresentadas as teorias relacionadas à abordagem deste trabalho, a fim de suportar o problema de pesquisa e pautar as hipóteses estudadas. Os próximos títulos aprofundam os conhecimentos sobre qualidade do planejamento, sucesso em projetos, risco em projetos e capacitação profissional, bem como as relações teóricas construídas entre as variáveis.

\subsection{Qualidade do planejamento}

O planejamento é um elemento central na gestão. Do mesmo modo, em gerenciamento de projetos, o planejamento é considerado um dos maiores colaboradores para o sucesso (Pinto \& Slevin, 1987). O planejamento do projeto especifica um conjunto de decisões dedicadas às formas que as atividades devem ser feitas no futuro no sentido de executar o idealizado para um produto ou serviço desejado (Zwikael \& Sadeh, 2007).

O PMBOK® contém um padrão global reconhecido para a profissão de gerenciamento de projetos. É um documento formal que estabelece normas, métodos e processos, gerado a partir de reconhecidas boas práticas de profissionais que contribuíram para o 
desenvolvimento (PMI, 2013; Varajão, Colomo-Palacios, \& Silva, 2017). Dos 47 processos apresentados pelo PMBOK®, 24 pertencem à fase de planejamento do projeto, abrangendo todas as 10 áreas de conhecimento (PMI, 2013). Por contemplar mais da metade dos processos de gerenciamento de projetos e compreender todas as áreas de conhecimento, a relevância do planejamento em projetos é evidente.

Zwikael e Globerson (2004) apresentaram e validaram uma escala de medida focada nos processos de planejamento, seguindo as boas práticas e cobrindo todas áreas de conhecimento mencionadas no PMBOK®. Para obtenção do índice chamado de Quality of Planning (QP), gerentes de projetos foram perguntados sobre a utilização desses processos, com objetivo de avaliar a frequência com que cada um é desenvolvido no projeto ou promovido pela organização. Outros autores seguiram o mesmo procedimento ao adotar o índice QP em seus trabalhos, como os estudos de Zwikael e Sadeh (2007) e Zwikael et al. (2014)

$\mathrm{O}$ índice QP consiste em duas partes: (1) Processos de conhecimento (know-how) do projeto (QPM), definida como a frequência com que os processos de planejamento são executados pelo gerente de projeto; e (2) Processos de suporte organizacional (QPO), definida como a frequência com que a organização se coloca e coloca meios à disposição do gerente de projetos para permitir o planejamento apropriado, execução e conclusão do mesmo (Zwikael \& Globerson, 2004; Zwikael \& Sadeh, 2007). Portanto, assume-se que os gerentes com os melhores resultados no índice QP planejam melhor os seus projetos.

\subsection{Sucesso em projetos}

Apesar dos estudos de sucesso organizacional terem se posicionado como o coração da teoria das organizações por muitos anos, a pesquisa sobre o sucesso do projeto não tem se direcionado para uma abordagem padrão (Dvir et al., 2003).

A literatura tem identificado a eficiência e a eficácia como duas importantes e distintas dimensões de sucesso do projeto (Shenhar \& Dvir, 2007). A eficiência do projeto se refere a alcançar os resultados esperados em tempo e custo, enquanto a eficácia se refere ao grau em que as especificações do projeto e as necessidades dos clientes são alcançados ou solucionados (Jugdev \& Müller, 2005; Zwikael et al., 2014). 
Zwikael e Sadeh (2007) trazem como medidas de sucesso: desempenho em custo, desempenho em tempo, desempenho técnico e satisfação do cliente. Enquanto desempenho em custo e em tempo são representados pelos desvios observados em relação ao planejado, o desempenho técnico é caracterizado como o cumprimento dos requisitos que fazem parte do escopo do projeto (Serrador \& Turner, 2014) e a satisfação do cliente é o senso que os clientes geram ao ter uma experiência na qual o serviço cumpre ou supera suas expectativas (Tae, Thai, \& Yeon, 2015). Zwikael et al. (2014) apresentam os mesmos elementos divididos em duas dimensões: eficiência (para as duas primeiras medidas) e eficácia (para as duas últimas medidas) em uma perspectiva mais objetiva para representar o sucesso do projeto, critério adotado nesta pesquisa.

\subsection{Risco em projetos}

Risco é definido como um evento ou condição incerta que, se ocorrer, provocará um efeito positivo ou negativo em um ou mais objetivos do projeto (Muriana \& Vizzini, 2017; PMI, 2013). Uma ferramenta simples, conveniente e utilizada para avaliação dos riscos é a matriz de probabilidade e impacto (PMI,
2013), onde as propriedades básicas "impacto" e "probabilidade" de um evento adverso são mostradas graficamente e mapeados por categorias, por meio de variáveis discretas (Duijm, 2015; Farooq, Thaheem, \& Arshad, 2018).

Apesar do risco não poder ser completamente eliminado, Chapman e Ward (2004) encontraram que as organizações dispendem valores significativos e recursos no gerenciamento de riscos. Alguns trabalhos se posicionam na corrente de pesquisa que incluí a visão baseada no risco e enfatizam a significância de gerenciar os riscos críticos (Liu, 2015; Liu, Zhang, Keil, \& Chen, 2010; Schmidt, Lyytinen, Keil, \& Cule, 2001; Wallace \& Keil, 2004). Entretanto, a literatura indica problemas recentes com as ferramentas de gestão de riscos dentro do ambiente de gerenciamento de projetos (Zwikael \& Sadeh, 2007).

Kerzner (2009) mostra a redução de incertezas como um motivo central para planejar um projeto. Zwikael e Sadeh (2007), em um estudo empírico com 202 gerentes de projetos de Israel, sugeriram o planejamento como meio para ser mais efetivo em projetos de alto risco do que em projetos de baixo risco, e concluem que os gerentes de projetos 
que informam estarem sujeitos a altos níveis de risco, estão relacionados a melhor qualidade de planejamento em seus projetos. Na mesma linha, Zwikael et al. (2014), em um estudo empírico com 183 projetos das Ilhas Fiji, destacam que a importância do planejamento depende do nível de risco que o projeto está sujeito, quando o sucesso é medido pela eficiência.

\subsection{Capacitação profissional}

O processo de capacitação profissional é definido como o desenvolvimento de conhecimento relacionado ao trabalho e às habilidades dos empregados, com objetivo de aumentar o desempenho sistematicamente (Swanson, 1999). O treinamento de extensão profissional contribui para desenvolver recursos humanos dentro dos limites da organização (Tabassi \& Bakar, 2009).

A literatura mostra uma forte relação entre conhecimentos ou habilidades com os resultados gerais da equipe do projeto (Liu \& Cross, 2016). No setor da construção, estudos indicam que a qualificação profissional afeta o desempenho geral do projeto (Odusami, Iyagba, \& Omirin, 2003) e que o investimento em capital humano promove melhorias no desempenho, confirmando que a educação e o treinamento são importantes e influenciam o tempo de entrega dos projetos (A. W. Brown et al., 2007). Em um estudo empírico realizado no Irã com profissionais da construção civil, os resultados indicaram que aproximadamente $56 \%$ dos projetos de construção tem baixa qualidade devido ao uso de trabalho não qualificado (Tabassi \& Bakar, 2009).

Capital humano é uma das áreas de pesquisa mais frequentes nas ciências econômicas nas últimas décadas e é marcada pela ideia de investir nas pessoas (Kozioł, Kozioł, Wojtowicz, \& Pyrek, 2014). Autores argumentam que a educação e os sistemas de treinamento devem fazer mais para preparar as equipes de projetos nas suas jornadas a fim de evoluir do bom para o ótimo (Ramazani \& Jergeas, 2015).

$\mathrm{O}$ estoque de capital humano pode ser mensurado como a média dos anos de escolaridade por pessoa ou trabalhador ativo na economia (Nakabashi, 2018; Nehru, Swanson, \& Dubey, 1995). A equação assume que trabalhadores sem formação não possuem capital humano e que o estoque de capital humano aumenta proporcionalmente com a duração da escolaridade (Bils \& Klenow, 2000; Lee 
\& Lee, 2016). Avaliar os atributos de conhecimento e habilidade de indivíduos e organizações é uma área abordada pela Teoria do Capital Humano, onde o foco é o valor que é adicionado ao negócio da organização pelo seu estoque de capital humano (A. W. Brown et al., 2007).

Capital humano geral é definido por conhecimentos genéricos e habilidades, não específicas para uma tarefa ou empresa, usualmente acumulada através de experiência profissional e formação, enquanto capital humano específico é usualmente acumulado através da formação e do treinamento em conhecimento específico para a empresa/tarefa (Au, Altman, \& Roussel, 2008; Kottaridi, Louloudi, \& Karkalakos, 2019).

Serão considerados neste trabalho profissionais capacitados aqueles que possuem cursos ou treinamentos específicos que os habilitam a executar as atribuições da sua respectiva função no projeto, de acordo com a compreensão do gerente responsável pelo projeto, excluindo da análise requisitos como o tempo de experiência no setor ou na função, não se limitando apenas ao gerente, incluindo na análise, dessa forma, profissionais de todos os níveis hierárquicos da equipe do projeto. Portanto, será avaliado o capital humano específico dos colaboradores na classificação do gerente.

\subsection{Relações teóricas e apresentação das hipóteses}

Estudos anteriores encontraram que a qualidade do planejamento tem um impacto positivo no sucesso do projeto (Pinto \& Slevin, 1987; Zwikael \& Globerson, 2004; Zwikael \& Sadeh, 2007), afetando diretamente a eficiência do projeto e a satisfação dos clientes (Shenhar \& Dvir, 2007; Zwikael et al., 2014), o que suporta a premissa de pesquisa que indica que os gerentes que planejam melhor obtém maior sucesso em seus projetos.

O gerente de projetos é responsável por concluir o projeto para a satisfação de todas as partes interessadas relevantes (Zwikael \& Globerson, 2006; Zwikael \& Sadeh, 2007). Para tal, esse profissional deve não apenas estar certo que as ações foram executadas de acordo com o plano, mas também assegurar que o plano é confiável e representa apropriadamente os requisitos das partes interessadas (Zwikael \& Globerson, 2006). Portanto, a percepção do gerente de projeto tem papel relevante na identificação e classificação dos riscos e da participação de profissionais capacitados. 
No sentido de aumentar as chances de sucesso do projeto, os gerentes de projetos são motivados a reduzir riscos a partir da implementação de melhor planejamento e controle (Zwikael \& Sadeh, 2007). A afirmação e toda a construção levam à reflexão que em projetos onde são percebidos maiores níveis de riscos, os gerentes são levados a dedicar maiores esforços ao planejamento. Enquanto isso, em projetos com menores níveis de risco percebidos, os esforços são direcionados para os demais processos em detrimento do planejamento. Portanto, pode-se sugerir que o nível de risco apresenta efeito de moderação na relação entre a qualidade do planejamento e as dimensões de sucesso do projeto, como indicado abaixo nas duas primeiras hipóteses de pesquisa.

Hipótese 1 (H1): O impacto da qualidade do planejamento na eficiência do projeto é maior à medida que o nível de risco percebido pelo gerente do projeto aumenta.

Hipótese 2 (H2): O impacto da qualidade do planejamento na eficácia do projeto é maior à medida que o nível de risco percebido pelo gerente do projeto aumenta.

Dentro desse entendimento, a literatura sugere que novas variáveis podem oferecer efeito moderador entre a qualidade do planejamento e o sucesso do projeto (Zwikael et al., 2014), fato que motiva a proposição de elementos ainda não explorados, entretanto suportados pela teoria e pela prática.

Diante da Teoria do Capital Humano e de um cenário recente onde o acesso ao conhecimento por meio de cursos de capacitação nas áreas de atuação possui grande disseminação, espera-se que a capacitação profissional dos colaboradores da equipe do projeto seja um elemento responsável por gerar cenários que alterem a intensidade da relação principal estudada.

Podemos esperar que quanto maior o estoque de capital humano de uma empresa, maior será o seu sucesso e a sua vantagem competitiva sobre os seus rivais, e vice-versa. A importância estratégica do capital humano em termos de alcançar aumento de desempenho está hoje se tornando cada vez mais reconhecida (Hitt, Bierman, Shimizu, \& Kochhar, 2001). Entretanto, apesar disso, um entendimento preciso de quão significante o papel do capital humano é em determinar o desempenho permanece não esclarecido e é o tema de muitas pesquisas em vários setores (Hitt et al., 2001; Laursen, Mahnke, \& VejrupHansen, 2003). 
Para buscar novos achados e esclarecer se os efeitos da capacitação profissional são suficientemente relevantes a ponto de compensar as vantagens proporcionadas pelo planejamento no contexto de gerenciamento de projetos, motivou-se a verificação das seguintes hipóteses de pesquisa.

Hipótese 3 (H3): O impacto da qualidade do planejamento na eficiência do projeto é menor à medida que a participação de profissionais capacitados na equipe do projeto aumenta.

Hipótese 4 (H4): O impacto da qualidade do planejamento na eficácia do projeto é menor à medida que a participação de profissionais capacitados na equipe do projeto aumenta.

\section{Procedimentos metodológicos}

A presente pesquisa fez uso da abordagem metodológica quantitativa, por meio da realização de um survey. As fontes de dados utilizadas na pesquisa foram as respostas dos gerentes de projetos do Brasil em atuação nos últimos anos, com foco nas experiências vivenciadas em um único projeto recentemente concluído. Optou-se por uma amostragem não probabilística por conveniência, uma vez que a pesquisa foi realizada para estudar a relação entre as variáveis, mais do que para estimar acuradamente os valores da população (Cozby, 2003).

Com o aplicativo Survey Monkey®, adotou-se o questionário estruturado para consolidar a coleta de dados, sobretudo por permitir a coleta de forma padronizada, conforme é exigido pela pesquisa quantitativa (Malhotra, 2011). A divulgação do convite para participação na pesquisa avançou pela rede social profissional LinkedIn, onde foi realizada busca por perfis pela localização (Brasil) e pelo cargo (gerente de projetos). Estima-se que foram enviados no período de coleta de dados 2000 convites para participação na pesquisa.

Foram contabilizadas 464 respostas recebidas, das quais 257 foram validadas, sendo as demais invalidadas por serem respostas (a) retidas na pergunta filtro, (b) iniciadas e não concluídas, (c) com inconsistência por critérios de respostas estabelecidos, ou (d) com padrão suspeito de resposta por excesso de repetições.

Após a fase inicial de caracterização dos respondentes, a próxima etapa de indagações do questionário foi adaptada a partir da escala validada para medição da qualidade do planejamento, conhecida 
como índice QP (Zwikael \& Globerson, 2004; Zwikael et al., 2014; Zwikael \& Sadeh, 2007), totalizando 34 questões em duas dimensões: 17 questões sobre o suporte organizacional em 4 indicadores, abrangendo sistemas, culturas, estrutura e escritório de projetos (QPO) e 17 questões sobre os produtos de planejamento de projetos distribuídos em 10 indicadores, que representam as áreas de conhecimento em gerenciamento de projetos (QPM) pelo PMBOK® (PMI, 2013), incluindo gerenciamento de stakeholders.

Para o conjunto de questões sobre QPO e QPM, foi solicitado que o respondente indicasse a frequência com que os processos da fase de planejamento foram utilizados, tomado como referência uma escala tipo Likert de 1 a 5 , em que 1 represente "quase nunca" e 5 represente "sempre". Os valores dos indicadores representados por mais de uma questão foram obtidos pela média das respostas relacionadas esses indicadores.

Com relação ao sucesso, foram utilizadas as dimensões de eficiência do projeto (EE) e de eficácia do projeto (EA), obtidas por meio de questões sobre desempenho em custo, desempenho em tempo, desempenho técnico e satisfação do cliente (Zwikael \& Sadeh, 2007). As perguntas relacionadas ao desempenho em custo e ao desempenho em tempo (EE) foram descritas em função do desvio percentual em relação ao planejamento inicial do projeto (Zwikael et al., 2014; Zwikael \& Sadeh, 2007), enquanto as perguntas relacionadas ao desempenho técnico e à satisfação do cliente (EA) foram ilustradas por uma escala tipo Likert de 1 a 5 , onde 1 é "péssimo" e 5 é "ótimo" (Zwikael et al., 2014).

O Nível de risco (R) foi medido como o risco percebido pelo gerente de projeto, sendo o mesmo convidado a estimar o modo que o projeto foi classificado na fase de iniciação, utilizando-se de uma escala tipo Likert de 5 pontos, onde 1 é "muito baixo" e 5 é "muito alto" em relação aos possíveis impactos identificados (Zwikael et al., 2014).

Medir o capital humano é uma tarefa complexa, e uma das possíveis formas de mensuração é considerar o percentual da população com determinado nível de formação concluída para comparação com outros grupos da população (Kottaridi et al., 2019). Neste trabalho, a partir de uma nova escala proposta visando quantificar a parcela de profissionais capacitados no projeto, foram solicitadas as estimativas: 
(a) do número total de colaboradores envolvidos no projeto e (b) do número total de colaboradores dos quais as respectivas funções no projeto dependem de cursos de qualificação, graduação ou especialização para que os mesmos exerçam as suas atividades. Ao dividirmos o número de colaboradores capacitados (b) pelo número total de colaboradores (a), obtemos o percentual de profissionais capacitados do projeto (PC), classificado no intervalo de $0 \%$ a $100 \%$.

Para a análise dos dados, foi utilizada a Modelagem de Equações Estruturais (Structural Equation Modeling), com base nos Mínimos Quadrados Parciais (Partial Least Squares), que é formada por um conjunto de modelos estatísticos que fazem uso de técnicas de análise fatorial e de regressão múltipla para explicar as relações multiníveis entre diversas variáveis (Hair Jr., Black, Babin, Anderson, \& Tatham, 2009). A modelagem permitiu verificar a validade e a confiabilidade dos dados coletados, as relações e os impactos, a partir de perguntas que representaram o efeito da causalidade na direção dos construtos para as medidas, com os indicadores associados a um mesmo construto altamente correlacionados, portanto, reflexivos (Hair Jr., Hult, Ringle, \& Sarstedt, 2014). Para viabilizar a análise, foi utilizado o software SmartPLS 3.0.

\section{Análise dos resultados}

As 257 respostas válidas foram divididas em projetos de diferentes setores da economia. A Tabela 1 mostra que as áreas mais expressivas foram de construção e informação ou comunicação, que representam juntas mais que dois terços do total da amostra. Em relação à experiência dos respondentes, mais que um terço dos gerentes de projetos indicaram mais que 10 anos de experiência na função. 


\section{Tabela 1}

Respondentes da Amostra por Setor da Economia

\begin{tabular}{lrr} 
Setores & Respondentes & \multicolumn{1}{c}{$\%$} \\
\hline 1. Construção (edifícios, infraestrutura ou serviços especializados para construção). & 89 & $34,63 \%$ \\
2. Informação ou comunicação (telecomunicações ou tecnologia da informação). & 84 & $32,68 \%$ \\
3. Atividades técnicas (arquitetura ou engenharia). & 25 & $9,73 \%$ \\
4. Atividades científicas (pesquisa ou desenvolvimento científico). & 8 & $3,11 \%$ \\
5. Indústria de transformação (manutenção ou instalação de máquinas ou & 25 & $9,73 \%$ \\
equipamentos). & 26 & $10,12 \%$ \\
6. Outros. & $\mathbf{2 5 7}$ & $\mathbf{1 0 0 , 0 0 \%}$ \\
\hline Total &
\end{tabular}

Fonte: Dados da pesquisa.

Com os respondentes divididos por setores da economia, os resultados da Figura 1 demonstraram que o segmento Informação ou comunicação possui a maior a média de profissionais capacitados da amostra, seguido de Atividades técnicas e Atividades científicas, enquanto Construção apresenta a menor média de profissionais capacitados, próximo à média da Indústria de transformação. As variações significativas entre os grupos são indícios que diferentes modalidades de projetos demandam diferentes percentuais de profissionais capacitados.

\section{Figura 1}

\section{Média de Percentual de Profissionais Capacitados por Setor da Economia}

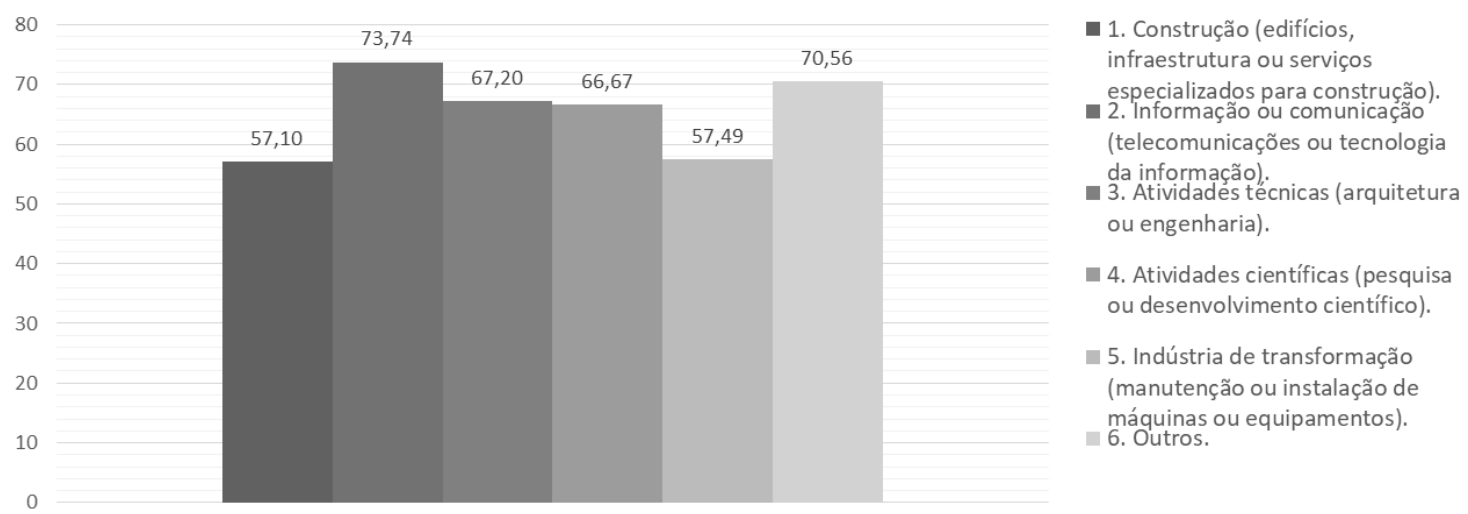

Fonte: Dados da pesquisa. 
Para a análise dos dados, foi utilizada a Modelagem de Equações Estruturais (Structural Equation Modeling), que é formada por um conjunto de modelos estatísticos que fazem uso de técnicas de análise fatorial e de regressão múltipla para explicar as relações multiníveis entre diversas variáveis (Hair Jr. et al., 2009). Para viabilizar a análise deste e dos próximos capítulos, foi utilizado o software SmartPLS 3.0 (Ringle, Wende, \& Becker, 2014), conforme indicado por Hair Jr. et al. (2014).

A validação do modelo de mensuração iniciou-se com os resultados gerados a partir dos construtos reflexivos de primeira ordem "QPM”, “QPO”, "Eficiência do projeto" e "Eficácia do projeto". Sem as moderações, o modelo convergiu com 7 iterações, muito inferior ao número máximo de 300 iterações.

Como é apresentado na Tabela 2, a consistência interna foi validada a partir de dois critérios recomendados por Hair Jr. et al. (2014): (1) confiabilidade composta, onde todos os valores entre 0,70 e 0,90 são considerados satisfatórios e definitivamente não devem ser superiores a 0,95; e (2) Alfa de Cronbach, onde sugerem-se valores superiores a 0,70. A validação da validade convergente deu-se pelas medidas do AVE (Average Variance Extracted), com os valores superiores a 0,50; e das cargas externas dos indicadores, com valores superiores a 0,70 ou entre 0,40 e 0,70 desde que a exclusão de um indicador afete a validade do conteúdo, aplicável aos indicadores qpm1, qpm2, qpm3 e qpm4 (Hair Jr. et al., 2014). 


\section{Tabela 2}

Validação de Consistência Interna e Validade Convergente Para os Construtos de Primeira

Ordem

\begin{tabular}{|c|c|c|c|c|c|}
\hline & & Consist & cia interna & Validad & ergente \\
\hline $\begin{array}{c}\text { Construtos de } \\
\text { primeira ordem }\end{array}$ & Indicadores & $\begin{array}{l}\text { Alfa de } \\
\text { Cronbach }\end{array}$ & $\begin{array}{c}\text { Confiabilidade } \\
\text { composta }\end{array}$ & $\begin{array}{c}\text { Carga } \\
\text { externa }\end{array}$ & AVE \\
\hline \multirow{10}{*}{ QPM } & qpm1 & \multirow{10}{*}{0,890} & \multirow{10}{*}{0,910} & 0,633 & \multirow{10}{*}{0,504} \\
\hline & qpm10 & & & 0,725 & \\
\hline & qpm2 & & & 0,653 & \\
\hline & qpm3 & & & 0,681 & \\
\hline & qpm4 & & & 0,631 & \\
\hline & qpm5 & & & 0,741 & \\
\hline & qpm6 & & & 0,753 & \\
\hline & qpm7 & & & 0,727 & \\
\hline & qpm8 & & & 0,764 & \\
\hline & qpm9 & & & 0,774 & \\
\hline \multirow{4}{*}{ QPO } & qpo1 & \multirow{4}{*}{0,881} & \multirow{4}{*}{0,918} & 0,816 & \multirow{4}{*}{0,738} \\
\hline & qpo2 & & & 0,898 & \\
\hline & qpo3 & & & 0,895 & \\
\hline & qpo4 & & & 0,824 & \\
\hline \multirow{2}{*}{$\begin{array}{l}\text { Eficiência do } \\
\text { projeto (EE) }\end{array}$} & sct & \multirow{2}{*}{0,740} & \multirow{2}{*}{0,883} & 0,916 & \multirow{2}{*}{0,791} \\
\hline & stp & & & 0,862 & \\
\hline \multirow{2}{*}{$\begin{array}{c}\text { Eficácia do projeto } \\
\text { (EA) }\end{array}$} & ssc & \multirow{2}{*}{0,704} & \multirow{2}{*}{0,871} & 0,882 & \multirow{2}{*}{0,772} \\
\hline & stc & & & 0,875 & \\
\hline
\end{tabular}

Fonte: Elaborado pelo autor (2019).

Com o objetivo de avaliar se um construto é realmente diferente dos demais construtos, dois testes são propostos por Hair Jr. et al. (2014) para análise da validade discriminante. Pelo critério de Fornell-Larcker foi comprovado que a raiz quadrada do AVE de cada construto é maior que a sua maior correlação com qualquer outro construto do modelo (Tabela 3). Pelo método das cargas cruzadas foi comprovado que as cargas externas dos indicadores associadas ao construto original são maiores que todas as suas demais cargas externas quando associadas aos demais construtos (Tabela 4). 


\section{Tabela 3}

Validação da Validade Discriminante Pelo Critério de Fornell-Larcker

\begin{tabular}{|c|c|c|c|c|}
\cline { 2 - 5 } \multicolumn{1}{c|}{} & EA & EE & QPM & QPO \\
\hline EA & 0,878 & & & \\
\hline EE & 0,277 & 0,890 & & \\
\hline QPM & 0,338 & 0,206 & 0,710 & \\
\hline QPO & 0,390 & 0,192 & 0,660 & 0,859 \\
\hline
\end{tabular}

Fonte: Elaborado pelo autor (2019).

\section{Tabela 4}

Validação da Validade Discriminante Pela Carga Cruzada dos Indicadores

\begin{tabular}{|c|c|c|c|c|c|}
\cline { 2 - 6 } \multicolumn{1}{c|}{} & Indicadores & QPM & QPO & EE & EA \\
\hline \multirow{6}{*}{ QPM } & qpm1 & 0,633 & 0,439 & $-0,169$ & 0,202 \\
& qpm10 & 0,725 & 0,499 & $-0,130$ & 0,190 \\
& qpm2 & 0,653 & 0,400 & $-0,119$ & 0,256 \\
& qpm3 & 0,681 & 0,404 & $-0,080$ & 0,283 \\
& qpm4 & 0,631 & 0,493 & $-0,216$ & 0,243 \\
& qpm5 & 0,741 & 0,505 & $-0,195$ & 0,283 \\
& qpm6 & 0,753 & 0,484 & $-0,162$ & 0,273 \\
& qpm7 & 0,727 & 0,482 & $-0,061$ & 0,272 \\
& qpm8 & 0,764 & 0,482 & $-0,118$ & 0,187 \\
& qpm9 & 0,774 & 0,486 & $-0,208$ & 0,216 \\
\hline \multirow{4}{*}{ QPO } & qpo1 & 0,492 & 0,816 & $-0,105$ & 0,321 \\
& qpo2 & 0,554 & 0,898 & $-0,159$ & 0,383 \\
& qpo3 & 0,656 & 0,895 & $-0,207$ & 0,302 \\
& qpo4 & 0,552 & 0,824 & $-0,181$ & 0,340 \\
\hline \multirow{2}{*}{ EE } & sct & 0,196 & 0,199 & 0,916 & 0,252 \\
& stp & 0,167 & 0,137 & 0,862 & 0,240 \\
\hline \multirow{2}{*}{ EA } & ssc & 0,319 & 0,316 & 0,290 & 0,882 \\
& stc & 0,274 & 0,371 & 0,195 & 0,875 \\
\hline
\end{tabular}

Fonte: Elaborado pelo autor (2019).

A partir da proposta de Hair Jr. et al. (2014) visando reduzir o número de relações no modelo estrutural e obter um modelo de caminho mais parcimonioso e de fácil entendimento, foi utilizado o construto de segunda ordem ou HOC (high-order component) denominado UP. O modelo de ordem superior ou HCM (high-order model ou hierarchical component model) criado é do tipo reflexivo-reflexivo, caracterizado pela relação entre o HOC UP e os construtos de primeira ordem ou LOC (low-order components) QPM e QPO. Para compor o HOC, foi adotada a abordagem dos indicadores repetidos.

A Tabela 5 mostra o resultado dos testes realizados para validação do 
construto de segunda ordem, a exemplo do que foi apresentado para os construtos de primeira ordem. Também a partir dos procedimentos e dos requisitos apontados por Hair Jr. et al. (2014), a consistência interna foi validada pela confiabilidade composta (valores entre 0,70 e 0,90 considerados satisfatórios e definitivamente não superiores a 0,95) e
Alfa de Cronbach (valores superiores a $0,70)$, enquanto a validade convergente deu-se pelas cargas externas dos indicadores (valores superiores a $0,70 \mathrm{ou}$ entre 0,40 e 0,70 desde que a exclusão do indicador afete a validade do conteúdo, aplicável aos indicadores qpm1, qpm2, qpm3, qpm4, qpm7, qpm10 e qpo1).

\section{Tabela 5}

Validação de Consistência Interna e Validade Convergente Para o Construto de Segunda Ordem

\begin{tabular}{|c|c|c|c|c|}
\hline \multirow[b]{2}{*}{$\begin{array}{c}\text { Construto de } \\
\text { segunda ordem }\end{array}$} & \multirow[b]{2}{*}{ Indicadores } & \multicolumn{2}{|c|}{ Consistência interna } & \multirow{2}{*}{$\begin{array}{c}\text { Validade convergente } \\
\text { Carga externa }\end{array}$} \\
\hline & & $\begin{array}{l}\text { Alfa de } \\
\text { Cronbach }\end{array}$ & $\begin{array}{c}\text { Confiabilidade } \\
\text { composta }\end{array}$ & \\
\hline \multirow{14}{*}{$\begin{array}{c}\text { Uso das técnicas de } \\
\text { planejamento }\end{array}$} & qpm1 & \multirow{14}{*}{0,916} & \multirow{14}{*}{0,928} & 0,613 \\
\hline & qpm10 & & & 0,698 \\
\hline & qpm2 & & & 0,611 \\
\hline & qpm3 & & & 0,632 \\
\hline & qpm4 & & & 0,635 \\
\hline & qpm5 & & & 0,714 \\
\hline & qpm6 & & & 0,713 \\
\hline & qpm7 & & & 0,693 \\
\hline & qpm8 & & & 0,718 \\
\hline & qpm9 & & & 0,728 \\
\hline & qpo1 & & & 0,667 \\
\hline & qpo2 & & & 0,742 \\
\hline & qpo3 & & & 0,810 \\
\hline & qpo4 & & & 0,711 \\
\hline
\end{tabular}

Fonte: Elaborado pelo autor (2019).

Para gerar os resultados da significância e da relevância dos coeficientes de caminho do modelo estrutural, foi utilizado o método Bootstraping. A Tabela 6 mostra que os quatro caminhos do modelo apresentam p-valor menores que 0,05 , o que representa que todas as relações do modelo são significantes a um nível adotado de $5 \%$. Ao comparar a relação da Qualidade do planejamento (QP) com as duas dimensões de sucesso, é possível 
identificar maior relevância da relação com a Eficácia do projeto (EA) em detrimento à Eficiência do projeto (EE). A Figura 2 ilustra as relações entre os construtos com a indicação dos coeficientes de caminho padronizados e $p$-valor (entre parênteses).

\section{Tabela 6}

Significância e Relevância dos Coeficientes de Caminho do Modelo Estrutural

\begin{tabular}{|c|c|c|c|c|c|}
\hline Caminhos & $\begin{array}{c}\text { Coeficiente } \\
\text { de caminho }\end{array}$ & $\begin{array}{c}\text { Média da } \\
\text { amostra }\end{array}$ & $\begin{array}{c}\text { Desvio } \\
\text { padrão }\end{array}$ & t-valor & p-valor \\
\hline $\mathrm{QP} \rightarrow \mathrm{EE}$ & 0,219 & 0,230 & 0,057 & 3,812 & 0.000 \\
\hline $\mathrm{QP} \rightarrow \mathrm{EA}$ & 0,390 & 0,395 & 0,059 & 6,654 & 0.000 \\
\hline $\mathrm{QP} \rightarrow \mathrm{QPM}$ & 0,954 & 0,954 & 0,006 & 155,177 & 0.000 \\
\hline $\mathrm{QP} \rightarrow \mathrm{QPO}$ & 0,855 & 0,857 & 0,018 & 48,865 & 0.000 \\
\hline
\end{tabular}

Fonte: Dados da pesquisa.

\section{Figura 2}

Coeficientes de Caminho no Modelo Estrutural com P-Valor

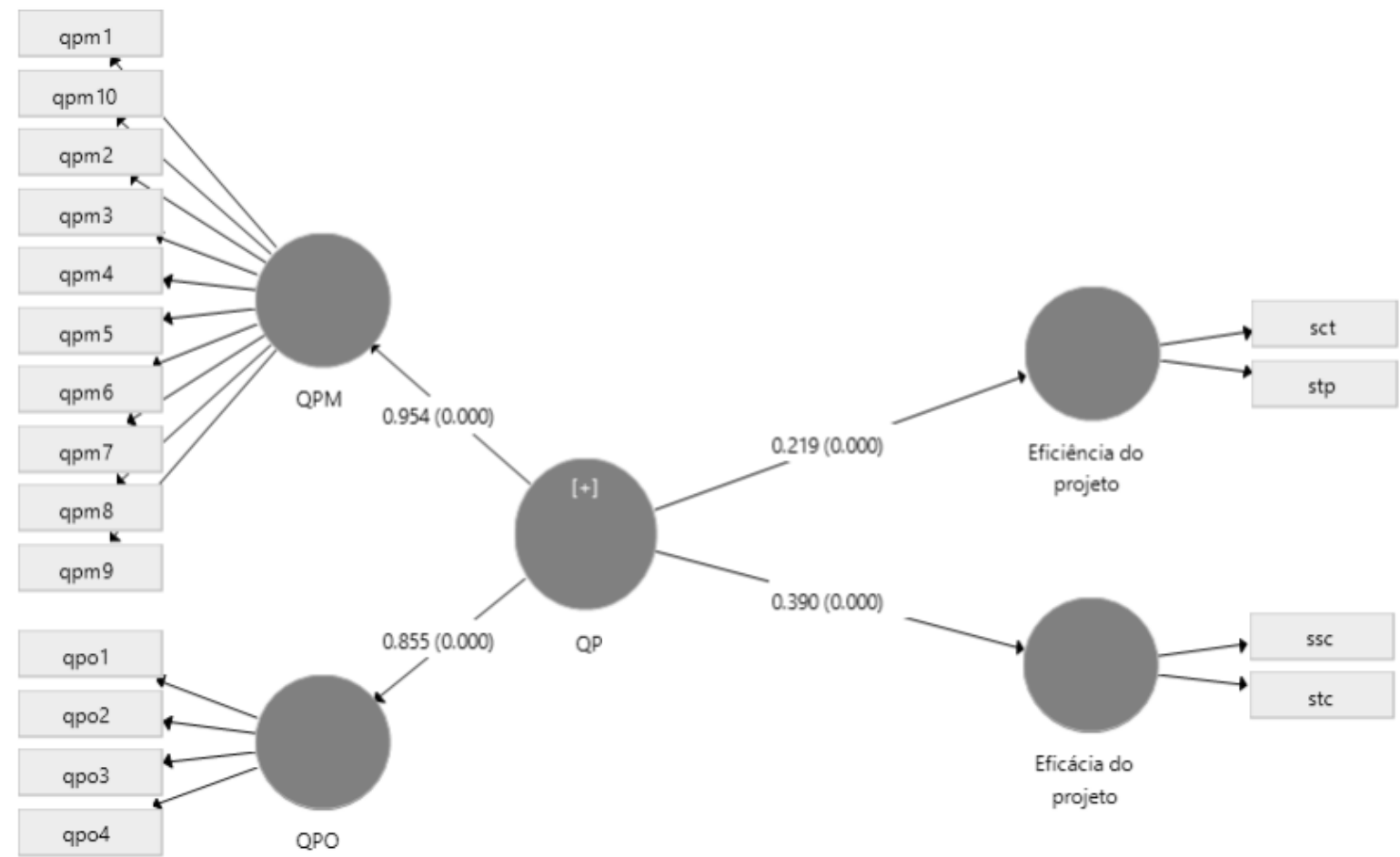

Fonte: Dados da pesquisa a partir do SmartPLS SEM 3.0.

Os dados levantados repetiram os resultados constatados nas pesquisas anteriores, onde o planejamento impacta no sucesso do projeto (Pinto \& Slevin, 
1987; Shenhar \& Dvir, 2007; Zwikael \&

Globerson, 2004; Zwikael et al., 2014;

Zwikael \& Sadeh, 2007). As relações da

Qualidade do planejamento com a

Eficiência do projeto $(\mathrm{QP} \rightarrow \mathrm{EE})$ e da

Qualidade do planejamento com a

Eficácia do projeto $(\mathrm{QP} \rightarrow \mathrm{EA})$

apresentaram significância estatística.

Esse resultado sugere que, ao adotarem técnicas de planejamento, as empresas aumentam as chances de alcançarem efeitos positivos em seus indicadores relacionados à eficácia e à eficiência do projeto. Portanto, foi cumprida a premissa para sequência do trabalho e permite-se que sejam avaliados os fatores que moderem as intensidades dessas relações.

\section{Reconhecendo que a} heterogeneidade na estrutura dos dados por meio de diferentes resultados nos coeficientes de caminho entre grupos de respondentes frequentemente está presente, os pesquisadores estão cada vez mais interessados em identificar e entender tais diferenças (Hair Jr. et al., 2014). Para testar as hipóteses da pesquisa pela moderação, foram incluídas no modelo as variáveis Nível de risco (R) e Participação dos profissionais capacitados no projeto (PC) ligadas às duas dimensões de sucesso (EE e EA), bem como os termos de interação de R e PC nas relações da Qualidade do planejamento (QP) com a Eficiência do projeto (EE) e com a Eficácia do projeto (EA):

- EE $\mathrm{R}^{*} \mathrm{QP}$ : efeito moderador de nível de risco na relação entre a qualidade do planejamento com a eficiência do projeto.

- EA R*QP: efeito moderador de nível de risco na relação entre a qualidade do planejamento com a eficácia do projeto.

- EE $\mathrm{PC}^{*} \mathrm{QP}:$ efeito moderador de participação de profissionais capacitados no projeto na relação entre a qualidade do planejamento com a eficiência do projeto.

- EA PC*QP: efeito moderador de participação de profissionais capacitados no projeto na relação entre a qualidade do planejamento com a eficácia do projeto.

Foi adotada abordagem do indicador do produto para avaliar a moderação na relação principal, uma vez que os efeitos moderadores são contínuos e são medidos usando construto de natureza reflexiva (Hair Jr. et al., 2014). Antes de serem gerados os resultados da significância e da relevância da moderação, os termos de interação foram submetidos aos critérios 
de validação aplicáveis aos construtos reflexivos. Para o modelo de mensuração foram verificadas a consistência interna e a validade convergente, enquanto em relação ao modelo estrutural foram verificadas as questões de colinearidade, a partir dos requisitos indicados por Hair Jr. et al. (2014).

Foi utilizado novamente o método Bootstraping para gerar os resultados de significância e relevância dos coeficientes de caminho, desta vez dos termos de interação. A Tabela 7 mostra que dos quatro caminhos que representam as moderações de $\mathrm{R}$ e PC nas relações de QP com EE e de QP com
EA, apenas uma apresentou p-valor menor que 0,05 , o que indica que de todas as relações do modelo a única moderação com significância estatística a um nível de $5 \%$ é do Nível de risco na relação da Qualidade do planejamento com a Eficiência do projeto, comprovando a hipótese $\mathrm{H} 1$ e rejeitando as demais $\mathrm{H} 2, \mathrm{H} 3$ e $\mathrm{H} 4$. A Figura 3 apresenta as relações entre os construtos com a indicação dos coeficientes de caminho padronizados e p-valor (entre parênteses), enquanto a Figura 4 ilustra significância dos caminhos por hipótese de pesquisa.

\section{Tabela 7}

Significância e Relevância dos Coeficientes de Caminho dos Termos de Interação

\begin{tabular}{|c|c|c|c|c|c|c|}
\hline Caminhos & Hipótese & $\begin{array}{c}\text { Coeficiente de } \\
\text { caminho }\end{array}$ & $\begin{array}{c}\text { Média da } \\
\text { amostra }\end{array}$ & $\begin{array}{c}\text { Desvio } \\
\text { padrão }\end{array}$ & t-valor & p-valor \\
\hline $\mathrm{EE} \mathrm{R} * \mathrm{QP} \rightarrow \mathrm{EE}$ & $\mathrm{H} 1$ & 0,167 & 0,201 & 0,061 & 2,748 & 0,006 \\
\hline $\mathrm{EA} \mathrm{R} * \mathrm{QP} \rightarrow \mathrm{EA}$ & $\mathrm{H} 2$ & 0,186 & 0,007 & 0,199 & 0,936 & 0,350 \\
\hline $\mathrm{EE} \mathrm{PC} \mathrm{QP}^{\mathrm{E}} \rightarrow \mathrm{EE}$ & $\mathrm{H} 3$ & 0,131 & 0,058 & 0,159 & 0,823 & 0,411 \\
\hline $\mathrm{EA} \mathrm{PC} * \mathrm{QP} \rightarrow \mathrm{EA}$ & $\mathrm{H} 4$ & $-0,150$ & $-0,020$ & 0,195 & 0,771 & 0,441 \\
\hline
\end{tabular}

Fonte: Dados da pesquisa. 


\section{Figura 3}

Coeficientes de Caminho no Modelo Estrutural com Moderação com P-Valor

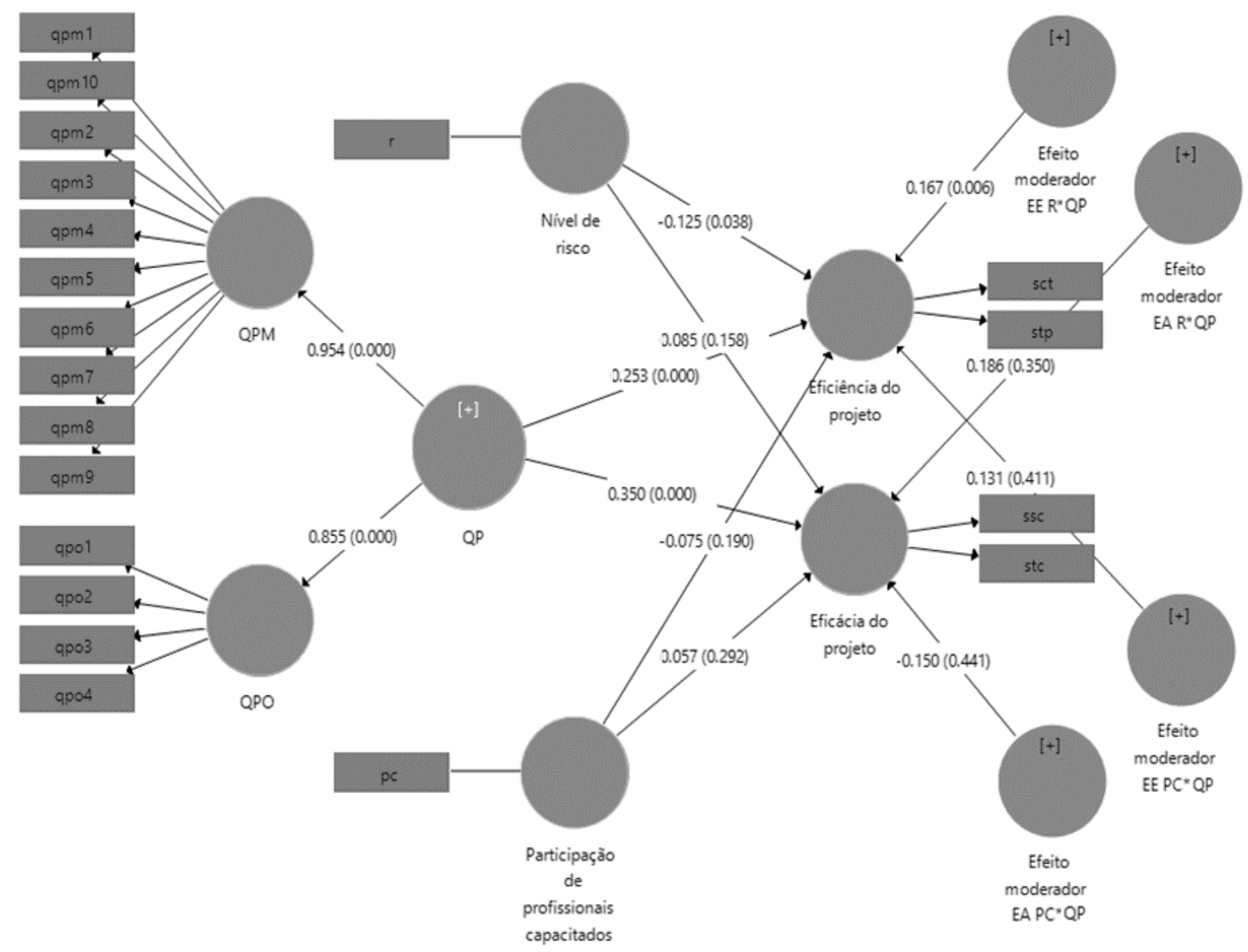

Fonte: Dados da pesquisa a partir do SmartPLS SEM 3.0. 


\section{Figura 4}

Modelo de Pesquisa e Hipóteses com Resultados

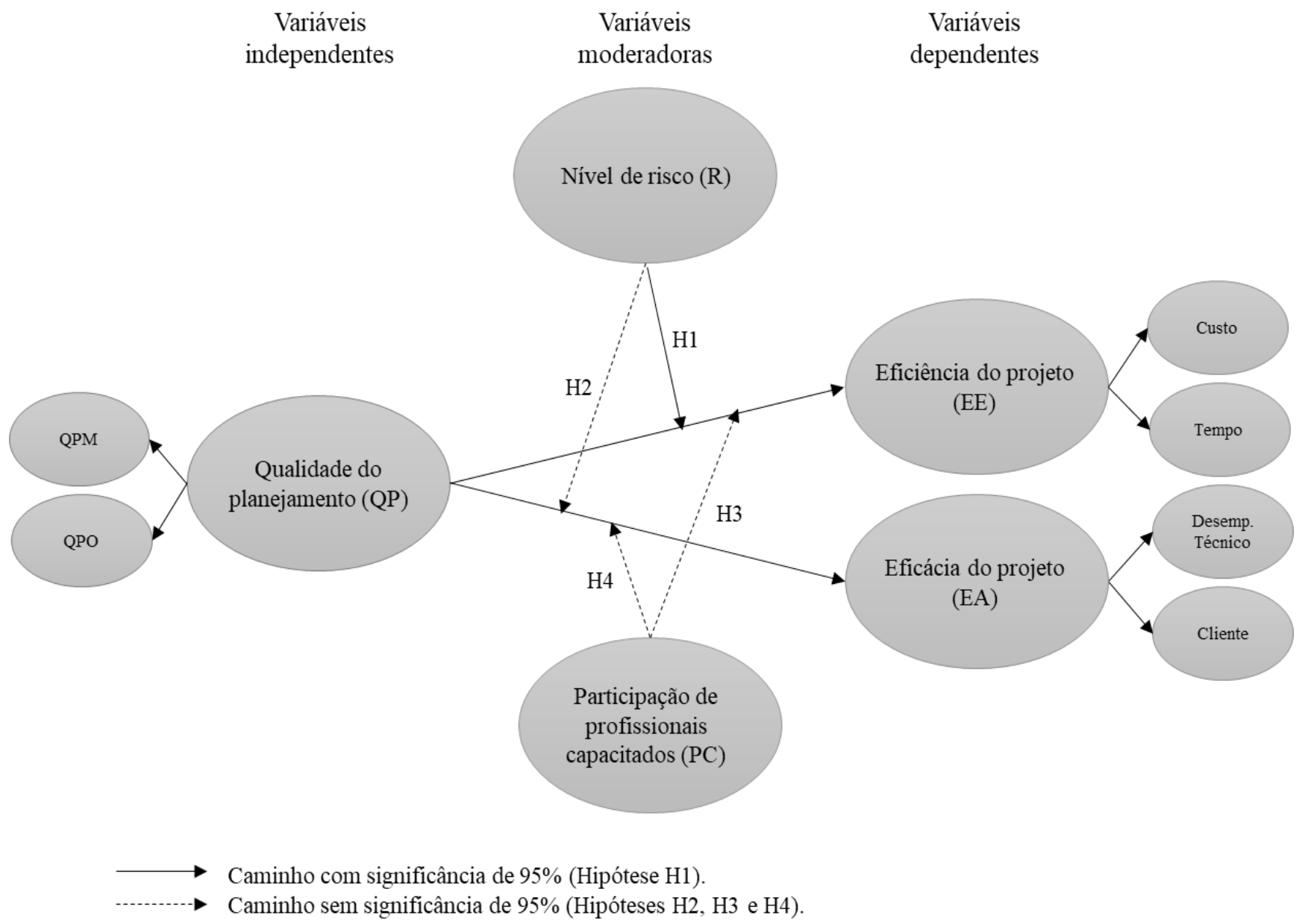

Fonte: Dados da pesquisa.

Por fim, foram testadas as relações diretas das variáveis latentes moderadoras Nível de risco (R) e Participação dos profissionais capacitados (PC) com os construtos endógenos Eficiência do projeto (EE) e
Eficácia do projeto (EA), como mostra a Tabela 8 e já representado na Figura 2. Das quatro combinações possíveis, apenas o caminho $\mathrm{R} \rightarrow \mathrm{EE}$ apresentou coeficiente com significância maior que $95 \%$. 


\section{Tabela 8}

Significância e Relevância dos Coeficientes de Caminho das Variáveis Moderadoras com os

Construtos Endógenos

\begin{tabular}{|c|c|c|c|c|c|}
\hline Caminhos & $\begin{array}{c}\text { Coeficiente } \\
\text { de caminho }\end{array}$ & $\begin{array}{c}\text { Média da } \\
\text { amostra }\end{array}$ & $\begin{array}{c}\text { Desvio } \\
\text { padrão }\end{array}$ & t-valor & p-valor \\
\hline $\mathrm{R} \rightarrow \mathrm{EE}$ & $-0,125$ & $-0,110$ & 0,060 & 2,080 & 0,038 \\
\hline $\mathrm{R} \rightarrow \mathrm{EA}$ & 0,085 & 0,060 & 0,060 & 1,414 & 0,158 \\
\hline $\mathrm{PC} \rightarrow \mathrm{EE}$ & $-0,075$ & $-0,073$ & 0,058 & 1,311 & 0,190 \\
\hline $\mathrm{PC} \rightarrow \mathrm{EA}$ & 0,057 & 0,076 & 0,054 & 1,054 & 0,292 \\
\hline
\end{tabular}

Fonte: Dados da pesquisa.

Ao testar a relação direta da variável Nível de risco (R) com as dimensões de sucesso Eficiência do projeto (EE) e Eficácia do projeto (EA), foi observado que apenas a relação $\mathrm{R} \rightarrow \mathrm{EE}$ apresentou $95 \%$ de significância estatística (p-valor 0,038). Ademais, o coeficiente de caminho obtido para essa relação foi negativo $(-0,125)$, o que indica que quanto maior é o nível de risco ao qual o projeto está submetido, menor será a eficiência do projeto. O resultado do risco provocando um efeito positivo ou negativo em um ou mais objetivos do projeto está alinhado com o sugerido no referencial teórico (Muriana \& Vizzini, 2017; PMI, 2013). Entretanto, enquanto esses achados são comprovados quanto aos desvios em tempo e custo (Eficiência do projeto), os mesmos comportamentos não puderam ser estendidos para o desempenho técnico e a satisfação do cliente (Eficácia do projeto), já que a relação apresentou significância estatística inferior a $95 \%$ (p-valor 0,158). Portanto, pode-se concluir que, na percepção do gerente de projeto do Brasil, o nível de risco ao qual o projeto está exposto não gera qualquer influência em seus resultados quanto à eficácia.

O impacto gerado pela Participação de profissionais capacitados (PC) não demonstrou significância quando relacionado à Eficiência do projeto (EE) (p-valor 0,190) ou à Eficácia do projeto (EA) (p-valor 0,292). Logo, possuir maior percentual de profissionais com capacitação não garante o sucesso do projeto em tempo, custo, desempenho técnico ou satisfação do cliente, que se explica por cada categoria de projeto possuir peculiaridades e diferentes demandas quanto à capacitação dos profissionais alocados para alcançar seus objetivos.

A variável latente Nível de risco (R) modera a relação entre a Qualidade do planejamento $(\mathrm{QP})$ e a Eficiência do projeto (EE), comprovada pela 
significância do seu coeficiente de caminho do termo de interação EE $\mathrm{R} * \mathrm{QP} \rightarrow \mathrm{EE}$ (com relevância de 0,167 , sentido positivo e p-valor 0,006), conforme indicado na Tabela 7. Essa constatação leva à conclusão de que, sob um cenário de maior percepção de exposição ao risco, maior será a dependência do planejamento para obter sucesso em tempo e custo. Em outra perspectiva, pode-se entender que utilizar técnicas de planejamento é um recurso adotado pelos gerentes de projetos como ferramenta de gestão de riscos, já que tornam-se evidentes em ambientes desfavoráveis como forma de mitigação dos mesmos, em concordância com as pesquisas publicadas sobre o tema quando aplicado o mesmo modelo em outros países (Zwikael et al., 2014; Zwikael \& Sadeh, 2007).

Contudo, os dados apontam que $\mathrm{R}$ não modera a relação entre $\mathrm{QP}$ e a dimensão de sucesso Eficácia do projeto (EA), resultado diferente dos achados na literatura aplicada a outros países por meio de outras bases metodológicas (Zwikael et al., 2014; Zwikael \& Sadeh, 2007), o que sugere que para diferentes níveis de risco envolvidos, não existirá alteração na influência do planejamento nessa dimensão de sucesso do projeto. Os achados indicam que o investimento em planejamento pelas empresas por si só não é suficiente para gerenciar riscos e proporcionar melhores retornos em desempenho técnico e satisfação do cliente no Brasil, diferença que pode ser explicada pela influência dos fatores locais na forma como os profissionais valorizam e adotam as atividades de planejamento ao gerenciar projetos (Bony, 2010; Bredillet, Yatim, \& Ruiz, 2010; Chevrier, 2003; Rodrigues, Costa, \& Guillén, 2014). Como destacado por Zwikael et al. (2014), a impossibilidade de generalizar os resultados passa pelos diferentes níveis de maturidade em gerenciamento de projetos que cada país apresenta.

Os resultados da Participação de profissionais capacitados (PC) como uma variável que não modera as relações entre a Qualidade do planejamento (QP) e sucesso (EE e EA) mostram que o amplo número de profissionais capacitados na equipe do projeto não substitui ou diminui a dependência do planejamento para alcançar o sucesso em eficiência ou eficácia. Desse modo, pode-se concluir que o fato de os profissionais possuírem capacitação para as atividades relacionadas às suas posições não é expressivo a ponto de dispensar (em parte ou completamente) os benefícios gerados ao utilizar as 
técnicas de planejamento, o que indica que apenas o conhecimento não é suficiente para substituir $\mathrm{o}$ ato de planejar e, portanto, considerar outros fatores como habilidades e atitude dos profissionais envolvidos podem ser determinantes para obter novos achados, pouco explorados em estudos anteriores.

Da Silva e Moraes (2021) estudaram como o capital humano atuou e foi impactado durante um cenário econômico complexo ou incerto e concluíram que esforços também relacionados à motivação e ao engajamento dos profissionais foram essenciais para superação do momento adverso sem maiores impactos.

Os achados indicam, portanto, que a Teoria do Capital Humano não se aplica integralmente às relações estudadas, ou seja, o estoque de capital humano aplicado apenas à capacitação profissional não gera valor ao negócio da organização no sentido de reduzir a necessidade de utilização do planejamento no projeto (A. W. Brown et al., 2007). Em perspectiva voltada para o papel do indivíduo e a sua relação com o capital, novas abordagens sugerem que a Teoria do Capital Humano ortodoxa não é adequada para os desafios encontrados no século XXI pela competição global, pelas novas tecnologias ou pelas desigualdades econômicas, propondo um pensamento alternativo no qual as pessoas não podem ser reduzidas ao que aprenderam ou ao seu estoque de conhecimento, ou seja, revalidando a forma como os profissionais entendem, utilizam e procuram se colocar no mercado de trabalho a partir de conhecimento e habilidades, por meio de um entendimento mais amplo de contribuições para a sociedade (P. Brown, Lauder, \& Cheung, 2020).

\section{Conclusão}

O presente estudo buscou, por meio de abordagem metodológica quantitativa e a partir das respostas de 257 gerentes de projetos no Brasil, analisar o efeito do nível de risco e da participação de profissionais capacitados na relação entre a qualidade do planejamento e o sucesso dos projetos no Brasil.

Da perspectiva teórica, este trabalho contribuiu ao compatibilizar o que literatura apresenta ao testar uma adaptação de um modelo validado por pesquisas anteriores em outras localidades agora dentro do contexto brasileiro, onde o nível de risco é moderador da relação entre 
planejamento e sucesso (Zwikael et al., 2014; Zwikael \& Sadeh, 2007).

Ainda do enfoque teórico, alinhado aos trabalhos já publicados sobre o tema, foi possível oportunizar refinamento de estudos anteriores e se dedicar a buscar novas variáveis moderadoras para a relação principal. Nesta pesquisa, foi testado o percentual de participação de profissionais capacitados como elemento moderador na relação entre planejamento e sucesso, o que proporcionou avanço para a teoria ao dar sequência aos trabalhos de Zwikael e Sadeh (2007) e de Zwikael et al. (2014), ainda que os dados não tenham suportado estatisticamente essa hipótese.

Dentre as implicações práticas, podemos deduzir que, uma vez comprovada a hipótese $\mathrm{H} 1$, aprimorar a qualidade do planejamento é uma maneira que as empresas brasileiras dispõem para mitigarem os riscos com potencial para impactar os objetivos em custo e prazos previstos dos seus projetos, apesar dessa prática não possuir a mesma função para aperfeiçoar o desempenho técnico e a satisfação do cliente (H2 rejeitada). Assim, os resultados mostram que o nível de risco altera a relação entre planejamento e eficiência, mas não entre planejamento e eficácia. O planejamento, sob condições de maior risco, pode ser determinante e é uma ferramenta utilizada para garantir resultados em termos de tempo e custo, mas não sofre interferência do nível de risco quando se considera o desempenho técnico e a satisfação do cliente, o que pode ser explicado pelo nível de maturidade em gerenciamento de projetos no local da pesquisa.

Por outro lado, as rejeições das hipóteses H3 e H4 nos levam a refletir que o fato de simplesmente possuir uma equipe de projeto formada por uma grande parcela de colaboradores com boa capacitação não reduz a dependência ou a responsabilidade da organização por planejar. Em outras palavras, investir apenas na capacitação profissional (conhecimento) da equipe envolvida, sem considerar também esforços nos demais fatores que suportam o capital humano (habilidade e atitude), não é suficiente para substituir os benefícios gerados pelo planejamento nos projetos.

Este trabalho apresenta algumas limitações usualmente inerentes às pesquisas cientificas. $\mathrm{O}$ modelo adotado, limitado a pesquisa à fase de planejamento do projeto, é uma adaptação de publicações anteriores que utilizam o Quality of Planning Index (índice QP), no qual cada uma das 10 
áreas de conhecimento em gerenciamento de projetos e cada um dos 4 grupos relacionados à estrutura organizacional possuem pesos iguais na formação do índice, sem qualquer diferenciação por ponderação (Zwikael \& Globerson, 2004; Zwikael et al., 2014; Zwikael \& Sadeh, 2007), o que pode ser melhor explorado, detalhado e revalidado, uma vez que grupos distintos podem possuir diferentes representatividades e, por consequência, diferentes pesos para compor esse indicador

Uma sugestão para estudos posteriores a partir deste trabalho é validação de novas variáveis moderadoras que representem elementos que alterem significativamente a relação entre planejamento e sucesso do projeto. Em relação à abordagem sobre o capital humano, pode-se propor identificar um percentual de profissionais capacitados na equipe do projeto de forma a otimizar o desempenho em eficiência ou eficácia por área da economia, bem como comprovar se a capacitação profissional combinada com novos fatores relacionados à equipe pode reduzir a dependência por planejar.

\section{Conflito de interesses}

Não houve conflitos.

\section{Contribuições de autoria}

Primeiro autor: elaboração do texto, objetivo, fundamentação teórica, método, resultados e conclusão.

Segundo autor: contribuição na definição de hipóteses, na análise dos resultados e na revisão do texto.

\section{Referências}

Au, A., Altman, Y., \& Roussel, J. (2008). Employee training needs and perceived value of training in the Pearl River Delta of China: A human capital development approach. Journal of European Industrial Training, 32(1), 19-31.

Bils, M., \& Klenow, P. (2000). Does Schooling Cause Growth? Does Schooling Cause Growth? and, (May 2014). Retrieved from https://doi.org/10.1257/aer.90.5. 1160

Bony, J. De. (2010). Project management and national culture : A Dutch - French case study. International Journal of Project Management, 28(2), 173-182. Retrieved from https://doi.org/10.1016/j.ijproma n.2009.09.002

Bredillet, C., Yatim, F., \& Ruiz, P. (2010). Project management deployment : The role of cultural factors. International Journal of Project Management, 28(2), 
183-193. Retrieved from https://doi.org/10.1016/j.ijproma n.2009.10.007

Brown, A. W., Adams, J. D., \& Amjad, A. A. (2007). The relationship between human capital and time performance in project management: A path analysis. International Journal of Project Management, 25(1), 77-89.

Retrieved from https://doi.org/10.1016/j.ijproma n.2006.07.011

Brown, P., Lauder, H., \& Cheung, S. Y. (2020). The death of human capital? Its failed promise and how to renew it in an age of disruption. New York: Oxford University Press. Retrieved from https://books.google.com.br/boo ks?hl=pt-

BR\&lr=\&id=SUD8DwAAQBA $\mathrm{J} \& \mathrm{oi}=\mathrm{fnd} \& \mathrm{pg}=\mathrm{PP} 1 \& \mathrm{dq}=\% 22 \mathrm{hu}$ man+capital+theory $\% 22 \&$ ots $=w$ q7v3PTpTW\&sig=Dey5jryxbidl sPNuM7bXxaa6Mug\#v=onepag $\mathrm{e} \& \mathrm{q} \& \mathrm{f}=$ true

Chapman, C., \& Ward, S. (2004). Why risk efficiency is a key aspect of best practice projects.

International Journal of Project Management, 22(8), 619-621.

Retrieved from

https://doi.org/10.1016/j.ijproma n.2004.05.001

Chevrier, S. (2003). Cross-cultural management in multinational project groups. Journal of World Business, 38, 141-149.

Retrieved from https://doi.org/10.1016/S10909516(03)00007-5

Cozby, P. C. (2003). Pesquisa de levantamento: uma metodologia para estimular pessoas a falar sobre si mesmas. In Métodos de pesquisa em ciências do comportamento (pp. 141-170). São Paulo: Atlas.

Da Silva, C. D. dos S., \& Moraes, A. B. G. de M. (2021). O papel estratégico do capital humano na gestão de crise - A pandemia de covid-19. Revista de Gestão e Projetos - (GeP), 12(2), 214232. Retrieved from https://doi.org/https://doi.org/10. 5585/gep.v12i2.19255

Duijm, N. J. (2015). Recommendations on the use and design of risk matrices. Safety Science, 76, 2131. Retrieved from https://doi.org/10.1016/j.ssci.201 5.02 .014

Dvir, D., Tzvi, R., \& Shenhar, A. J. (2003). An Empirical Analysis of the Relationship between Project Planning and Project Success project planning and project success. International Journal of Project Management, 21(2), 89-95. Retrieved from https://doi.org/10.1016/S02637863(02)00012-1

Farooq, M. U., Thaheem, M. J., \& Arshad, H. (2018). Improving the risk quantification under behavioural tendencies: A tale of construction projects, 36 , 414-428. Retrieved from https://doi.org/10.1016/j.ijproma n.2017.12.004

Hair Jr., J. F., Black, W. C., Babin, B. J., Anderson, R. E., \& Tatham, R. L. (2009). Análise Multivariada de Dados (6 $\left.{ }^{\mathrm{a}}\right)$. Porto Alegre: Bookman. 
Hair Jr., J. F., Hult, T. M., Ringle, C. M., \& Sarstedt, M. (2014). Partial Least Squares Structural Equation Modeling. Handbook of Market Research (Vol. 26). Los Angeles: Sage Publications. Retrieved from https://doi.org/10.1007/978-3319-05542-8_15-1

Hitt, M. A., Bierman, L., Shimizu, K., \& Kochhar, R. (2001). Direct and moderating effects of human capital on strategy and performance in professional service firms: A resource-based perspective. Academy of Management Journal, 44(1), 1328. Retrieved from https://www.scopus.com/record/ display.uri?eid=2-s2.00035255844\&origin=inward\&tx Gid=e14c9d3d99af44deaf7697f7 08e44aff

Jugdev, K., \& Müller, R. (2005). A retrospective look at our evolving understanding of project success. Project Management Journal, 36(4), 1931.

Kerzner, H. (2009). Project management: a systems approach to planning, scheduling, and conrolling (10th ed.). New York: John Wiley \& Sons, Inc.

Khan, A. S., \& Rasheed, F. (2015). Human resource management practices and project success, a moderating role of Islamic Work Ethics in Pakistani project-based organizations. International Journal of Project Management, 33(2), 435-445. Retrieved from https://doi.org/10.1016/j.ijproma n.2014.08.006
Kottaridi, C., Louloudi, K., \& Karkalakos, S. (2019). Human capital, skills and competencies : Varying effects on inward FDI in the EU context. International Business Review, 28(2), 375-390.

Retrieved from https://doi.org/10.1016/j.ibusrev. 2018.10.008

Kozioł, L., Kozioł, W., Wojtowicz, A., \& Pyrek, R. (2014). An Outline of a Compensation System Based on Human Capital Theory. Procedia - Social and Behavioral Sciences, 148, 551558. Retrieved from https://doi.org/10.1016/j.sbspro. 2014.07.078

Laursen, K., Mahnke, V., \& VejrupHansen, P. (2003). Do differences make a difference? The impact of human capital diversity and specificity on firm performance in engineering consulting. In DRUID Summer Conference 2003 (pp. 1-25). Copenhagen: DRUID.

Lee-kelley, L., \& Blackman, D. (2012). Project training evaluation: Reshaping boundary objects and assumptions. International Journal of Project Management, 30(1), 73-82. Retrieved from https://doi.org/10.1016/j.ijproma n.2011.02.010

Lee, J., \& Lee, H. (2016). Human capital in the long run. Journal of Development Economics, 122, 147-169. Retrieved from https://doi.org/10.1016/j.jdeveco .2016.05.006 
Liu, S. (2015). Effects of control on the performance of information systems projects: The moderating role of complexity risk. Journal of Operations Management, 36, 46-62. Retrieved from https://doi.org/10.1016/j.jom.201 5.03 .003

Liu, S., Zhang, J., Keil, M., \& Chen, T. (2010). Comparing senior executive and project manager perceptions of IT project risk : a Chinese Delphi study. Information Systems Journal, 20(4), 319-355. Retrieved from https://doi.org/10.1111/j.13652575.2009.00333.x

Liu, W. H., \& Cross, J. A. (2016). A comprehensive model of project team technical performance. International Journal of Project Management, 34(7), 1150-1166. Retrieved from https://doi.org/10.1016/j.ijproma n.2016.05.011

Malhotra, N. K. (2011). Pesquisa de marketing: uma orientação aplicada $\left(6^{\mathrm{a}}\right)$. Porto Alegre: Bookman.

Muriana, C., \& Vizzini, G. (2017). Project risk management: A deterministic quantitative technique for assessment and mitigation. International Journal of Project Management, 35(3), 320-340. Retrieved from https://doi.org/10.1016/j.ijproma n.2017.01.010

Nakabashi, L. (2018). Poverty and economic development : Evidence for the Brazilian states. EconomiA, 19(3), 445-458. Retrieved from https://doi.org/10.1016/j.econ.20 18.11.002

Nehru, V., Swanson, E., \& Dubey, A. (1995). A new database on human capital stock in developing and industrial countries: Sources, methodology, and results. Journal of Development Economics, 46, 379-401.

Odusami, K. T., Iyagba, R. R. O., \& Omirin, M. M. (2003). The relationship between project leadership, team composition and construction project performance in Nigeria. International Journal of Project Management, 21(7), 519-527.

Retrieved from https://doi.org/10.1016/S02637863(02)00059-5

Pinto, J. K., \& Slevin, D. P. (1987).

Critical Factors in Successful Project Implementation. IEEE Transactions on Engineering Management, 34(1), 22-27. Retrieved from https://doi.org/10.1109/TEM.19 87.6498856

PMI. (2013). A Guide to the Project Management Body of Knowledge (PMBOK® Guide) (5th ed.). Newtown Square, PA, USA: Project Management Institute. Retrieved from http://pmfiles.com/sites/default/files/file/ $\mathrm{C} / \mathrm{C}-1 / \mathrm{C}-1-$ 1/pmbok_5th_2013_eng.pdf

Ramazani, J., \& Jergeas, G. (2015). Project managers and the journey from good to great: The bene fi ts of investment in project management training and 
education. International Journal of Project Management, 33(1), 41-52. Retrieved from https://doi.org/10.1016/j.ijproma n.2014.03.012

Ringle, C. M., Wende, S., \& Becker, J.M. (2014). SmartPLS 3. Hamburg, Germany: SmartPLS.

Rodrigues, J. S., Costa, A. R., \& Guillén, C. (2014). Project planning and control: Does national culture influence project success ? Procedia Technology, 16, 1047-1056. Retrieved from https://doi.org/10.1016/j.protcy.2 014.10 .059

Schmidt, R. O. Y., Lyytinen, K., Keil, M., \& Cule, P. (2001). Identifying Software Project Risks : An International Delphi Study. Journal of Management Information Systems, 17(4), 536.

Serrador, P., \& Turner, J. R. (2014). The Relationship between Project Success and Project Efficiency. Social and Behavioral Sciences, 119, 7584.

Shenhar, A., \& Dvir, D. (2007). Project management research--the challenge and opportunity. Project Management Journal, 38(2), 93-99. Retrieved from https://www.pmi.org/learning/lib rary/project-managementresearch-challenge-opportunity5565

Swanson, R. A. (1999). HRD theory, real or imagined? Human Resource Development International, 2(1), 2-5. Retrieved from https://doi.org/10.1080/1367886 9900000002

Tabassi, A. A., \& Bakar, A. H. A. (2009). Training, motivation, and performance: The case of human resource management in construction projects in Mashhad, Iran. International Journal of Project Management, 27(5), 471-480. Retrieved from https://doi.org/10.1016/j.ijproma n.2008.08.002

Tae, G., Thai, V. V, \& Yeon, S. (2015). The Asian Journal of Shipping and Logistics An Analysis of Port Service Quality and Customer Satisfaction: The Case of Korean Container Ports. The Asian Journal of Shipping and Logistics, 31(4), 437-447. Retrieved from https://doi.org/10.1016/j.ajs1.201 6.01 .002

Varajão, J., Colomo-Palacios, R., \& Silva, H. (2017). ISO 21500:2012 and PMBoK 5 processes in information systems project management. Computer Standards and Interfaces, 50(May 2016), 216-222.

Retrieved from https://doi.org/10.1016/j.csi.201 6.09 .007

Wallace, L., \& Keil, M. (2004). How Software Project Risk Affects Project Performance : An Investigation of the Dimensions of Risk and an Exploratory Model *. Decision Sciences, 35(2), 289-321.

Zwikael, O., \& Globerson, S. (2004). Evaluating the quality of project planning: A model and field results. International Journal of 
Production Research, 42(8), 1545-1556. Retrieved from https://doi.org/10.1080/0020754 0310001639955

Zwikael, O., \& Globerson, S. (2006).

From Critical Success Factors to Critical Success Processes. International Journal of Production Research, 44(17), 3433-3449.

Zwikael, O., Pathak, R. D., Singh, G., \& Ahmed, S. (2014). The moderating effect of risk on the relationship between planning and success. International Journal of Project Management, 32(3), 435-441. Retrieved from https://doi.org/10.1016/j.ijproma n.2013.07.002

Zwikael, O., \& Sadeh, A. (2007). Planning effort as an effective risk management tool. Journal of Operations Management, 25(4), 755-767. Retrieved from https://doi.org/10.1016/j.jom.200 6.12.001 


\section{Apêndice}

Média eDesvio Padrão por Pergunta do Questionário

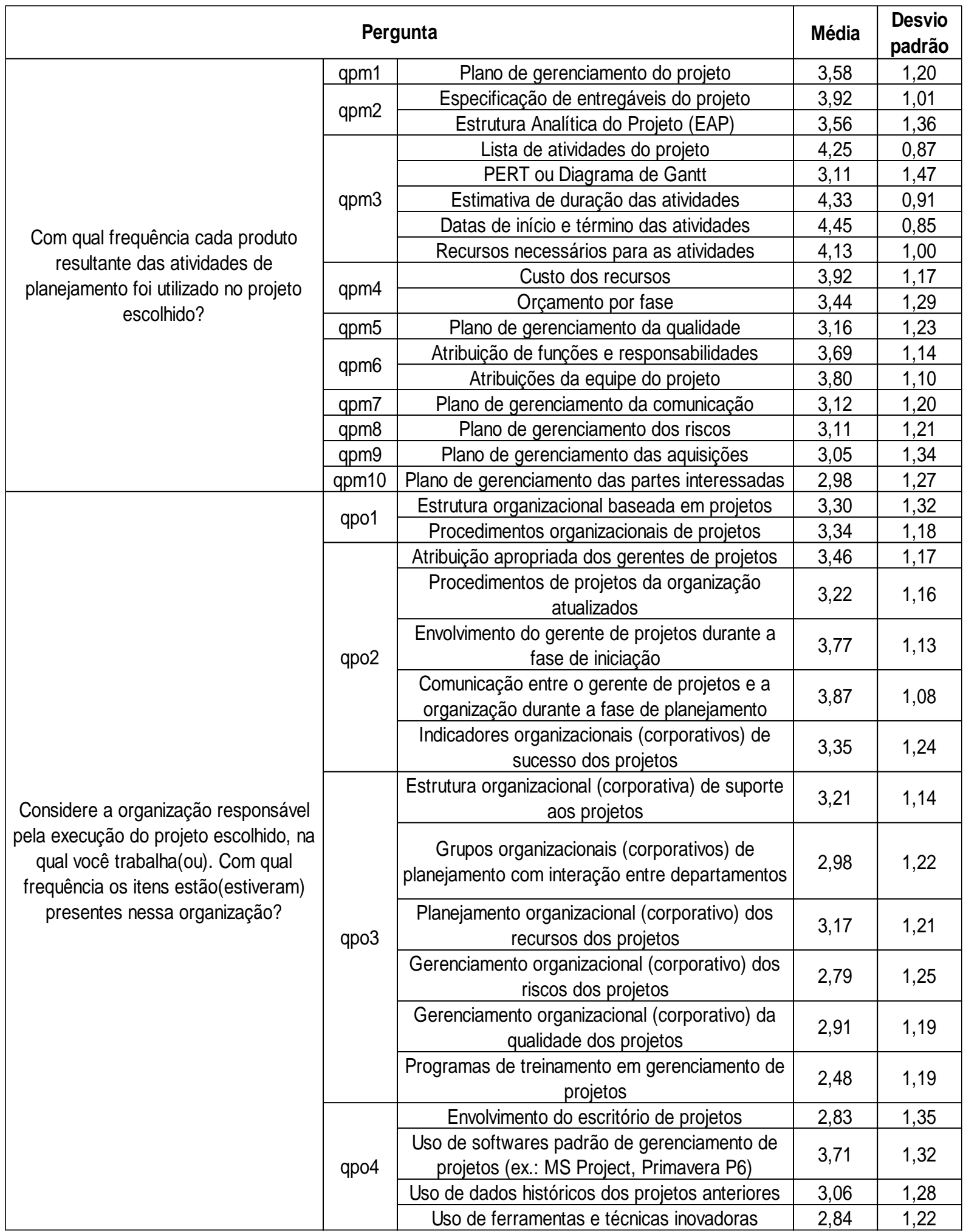


Roubach, L. B., Oliveira, M. P. V. (2021, maio/ago.). A relação entre o planejamento e o sucesso do projeto sob os efeitos do risco e da capacitação profissional

\begin{tabular}{|c|c|c|c|c|}
\hline \multicolumn{3}{|c|}{ Pergunta } & \multirow{2}{*}{$\begin{array}{c}\text { Média } \\
3,99\end{array}$} & \multirow{2}{*}{$\begin{array}{c}\begin{array}{c}\text { Desvio } \\
\text { padrão }\end{array} \\
22,86\end{array}$} \\
\hline $\begin{array}{l}\text { Qual foi o desvio percentual de custo } \\
\text { em relação ao planejado? }\end{array}$ & sct & Desvio de custo & & \\
\hline $\begin{array}{l}\text { Qual foi o desvio percentual de tempo } \\
\text { em relação ao planejado? }\end{array}$ & stp & Desvio de tempo & 11,34 & 25,34 \\
\hline \multirow{2}{*}{$\begin{array}{l}\text { Qual foi a sua avaliação do resultado } \\
\text { do projeto escolhido em relação aos }\end{array}$} & stc & Desempenho técnico & 4,09 & 0,68 \\
\hline & SSC & Satisfação do cliente & 4,15 & 0,73 \\
\hline $\begin{array}{l}\text { Qual foi o nível de risco percebido no } \\
\text { início de sua participação no projeto } \\
\text { escolhido em relação aos possíveis } \\
\text { impactos no cumprimento dos objetivos } \\
\text { do mesmo? }\end{array}$ & $r$ & Risco percebido & 3,32 & 0,89 \\
\hline \multirow[b]{2}{*}{$\begin{array}{l}\text { Considerando o pico das atividades do } \\
\text { projeto escolhido: }\end{array}$} & \multirow[b]{2}{*}{$\mathrm{pc}$} & $\begin{array}{l}\text { Qual foi o número total de profissionais } \\
\text { necessários na equipe do projeto para execução } \\
\text { do mesmo? }\end{array}$ & 255,38 & $1.150,84$ \\
\hline & & $\begin{array}{l}\text { Do número de profissionais necessários na } \\
\text { equipe do projeto para execução do mesmo, } \\
\text { quantos possuem cursos ou treinamentos } \\
\text { específicos para desempenhar as suas } \\
\text { respectivas funções, em quaisquer que sejam os } \\
\text { níveis hierárquicos? }\end{array}$ & 106,69 & 436,81 \\
\hline
\end{tabular}

Fonte: Dados da pesquisa. 